\title{
Systematic Environmental Evaluations To Identify Food Safety Differences between Outbreak and Nonoutbreak Restaurants
}

\author{
CRAIG W. HEDBERG, ${ }^{*}$ S. JAY SMITH, ${ }^{2}$ ELIZABETH KIRKLAND, ${ }^{2}$ VINCENT RADKE, ${ }^{2}$ TIM F. JONES, ${ }^{3}$ \\ CAROL A. SELMAN, ${ }^{2}$ AND THE EHS-NET WORKING GROUP
}

\begin{abstract}
${ }^{1}$ Division of Environmental Health Sciences, University of Minnesota, 420 Delaware Street S.E., Minneapolis, Minnesota 55455; ${ }^{2}$ National Center for Environmental Health, Centers for Disease Control and Prevention, 4770 Buford Highway, Atlanta, Georgia 30341; and ${ }^{3}$ Tennessee Department of Health, Cordell Hull Building, 425 5th Avenue N., 4th Floor, Nashville, Tennessee 37247, USA
\end{abstract}

MS 05-584: Received 15 November 2005/Accepted 28 March 2006

\begin{abstract}
Restaurants are important settings for foodborne disease transmission. The Environmental Health Specialists Network (EHS-Net) was established to identify underlying factors contributing to disease outbreaks and to translate those findings into improved prevention efforts. From June 2002 through June 2003, EHS-Net conducted systematic environmental evaluations in 22 restaurants in which outbreaks had occurred and 347 restaurants in which outbreaks had not occurred. Norovirus was the most common foodborne disease agent identified, accounting for $42 \%$ of all confirmed foodborne outbreaks during the study period. Handling of food by an infected person or carrier (65\%) and bare-hand contact with food (35\%) were the most commonly identified contributing factors. Outbreak and nonoutbreak restaurants were similar with respect to many characteristics. The major difference was in the presence of a certified kitchen manager (CKM); 32\% of outbreak restaurants had a CKM, but $71 \%$ of nonoutbreak restaurants had a CKM (odds ratio of $0.2 ; 95 \%$ confidence interval of 0.1 to 0.5 ). CKMs were associated with the absence of bare-hand contact with foods as a contributing factor, fewer norovirus outbreaks, and the absence of outbreaks associated with Clostridium perfringens. However, neither the presence of a CKM nor the presence of policies regarding employee health significantly affected the identification of an infected person or carrier as a contributing factor. These findings suggest a lack of effective monitoring of employee illness or a lack of commitment to enforcing policies regarding ill food workers. Food safety certification of kitchen managers appears to be an important outbreak prevention measure, and managing food worker illnesses should be emphasized during food safety training programs.
\end{abstract}

Foodborne illness remains an important public health problem in the United States (11), and restaurants are the most commonly identified setting for foodborne outbreaks (14). From 1993 through 1997, 45\% of confirmed foodborne outbreaks reported to the Centers for Disease Control and Prevention (CDC) occurred in restaurants (14). Although only a small proportion of foodborne illnesses are associated with outbreaks, four separate case-control studies conducted by FoodNet revealed an association between eating foods outside the home and an increased risk for specific foodborne illnesses (7). Thus, eating in restaurants appears to be an important general risk factor for foodborne disease transmission in the United States. Given that $80 \%$ of Americans eat out at least once per week, a better understanding of how and why transmission of foodborne diseases occurs in restaurants is needed to develop better prevention measures (7).

The Environmental Health Specialists Network (EHSNet) was established by the CDC in collaboration with the U.S. Food and Drug Administration and the U.S. Department of Agriculture to identify both how and why foodborne illness and disease outbreaks occur in food service settings and to translate those findings into improved prevention efforts using a systems-based approach $(3,5)$. EHS-

* Author for correspondence. Tel: 612-626-4757; Fax: 612-626-0650; E-mail: hedbe005@umn.edu.
Net was developed as a collaborative project with FoodNet sites in California, Colorado, Connecticut, Georgia, Minnesota, New York, Oregon, and Tennessee. Its initial purpose was to bring together environmental health specialists with retail food experience, epidemiologists, and laboratory scientists to learn about the causes of restaurant-associated foodborne illness. The purpose of the present study was to compare food handling practices and characteristics in outbreak and nonoutbreak restaurants to identify differences that have implications for food safety.

\section{MATERIALS AND METHODS}

Existing instruments used to assess the environmental conditions in restaurants measure food handling and sanitation deficiencies, regardless of whether the information is to be used for a routine inspection or an outbreak evaluation. Because these instruments do not capture the detailed data necessary to understand the how and why of foodborne disease outbreaks, an instrument and method were developed that could be used in restaurants in which foodborne illness outbreaks did and did not occur. Data collection instruments were developed to assess establishment characteristics, environmental conditions, and sanitation practices and to document individual food flows. Types of data collected included food handling policies and practices, languages spoken by food workers, training and certification, and staffing. Each of these instruments was extensively field tested in both outbreak and nonoutbreak restaurants and other foodservice settings during pilot studies. 
To learn more about food handling practices and how they relate to foodborne illness, we designed the study to conduct similar comprehensive evaluations and compare restaurants in which outbreaks did and did not occur. Outbreaks were defined as a foodborne illness involving two or more people who ate a common meal in a foodservice setting, with no other plausible common exposure.

For the outbreak restaurant evaluations, eligible establishments included all restaurants experiencing outbreaks reported within study sites during the study time frame. Because of differences in the relationships between individual EHS-Net sites and the food regulatory programs in their states, outbreak evaluations were conducted a median of 7 days after the initial outbreak investigation (range, 0 to 47 days). Of 107 eligible restaurant outbreaks that occurred in the EHS-Net sites during the study time frame, evaluations were completed for 22, primarily because of limited availability of EHS-Net specialists at the time of the outbreak. A median of three outbreaks were evaluated per state (range, 0 to 6). Outbreak investigation report forms submitted by the investigating agency (the CDC Electronic Foodborne Outbreak Reporting System [EFORS]) were reviewed to identify recognized contributing factors and were validated by the EHS-Net outbreak evaluations.

For nonoutbreak restaurant evaluations, eligible establishments included all restaurants within the study site that had no known history of foodborne outbreaks during the previous 3 years and no food-related illness complaints logged against them within the past year. Each site was asked to conduct 50 nonoutbreak restaurant evaluations over the study period. Outbreaks reported within the EHS-Net sites in the year prior to the study were reviewed to determine food service characteristics of outbreak establishments. The meal service types were as follows: $9 \%$ were prep serve (only ready-to-eat food items were prepared and served), $22 \%$ were cook serve (items were cooked to order for service), and 69\% were complex (food preparation included cooking, cooling, and reheating). A stratified random sample of restaurants was chosen to provide a similar distribution of nonoutbreak restaurants for comparison. Each site was asked to include 5 to 7 prep serve establishments, 10 to 15 cook serve establishments, and 30 to 35 complex establishments among their total of 50 nonoutbreak restaurant evaluations. However, because individual sites differed with respect to the organization of their food programs and restaurant license databases, specific enrollment procedures also differed among sites.

Data were analyzed to evaluate associations between restaurant characteristics and the occurrence of an outbreak. Thus, for most analyses, outbreak status was the outcome variable, and individual restaurant characteristics, such as type of ownership, manager training, policies, and evaluation results, served as predictor variables. Categorical univariate analyses with calculation of odds ratios (ORs), 95\% confidence intervals (CIs), and chisquare and Fisher's exact tests were conducted using Epiinfo, version 3.2.2 (2004, CDC, Atlanta, Ga.).

\section{RESULTS}

From 1 June 2002 through 30 June 2003, 179 foodborne outbreaks were confirmed in the EHS-Net catchment area and were reported to EFORS. Of these outbreaks, 107 $(60 \%)$ occurred in restaurants. EHS-Net specialists evaluated $22(21 \%)$ of the restaurant outbreaks (Table 1). Norovirus was the confirmed or suspected agent for $42 \%$ of all restaurant outbreaks and $45 \%$ of the evaluated outbreaks. Salmonella and Clostridium perfringens were the
TABLE 1. Confirmed foodborne outbreaks in restaurants and EHS-Net outbreak evaluations by agent, June 2002 through June 2003

\begin{tabular}{lcc}
\hline $\begin{array}{c}\text { Agent } \\
\text { (confirmed or suspected) }\end{array}$ & $\begin{array}{c}\text { No. (\%) of } \\
\text { outbreaks }^{a}\end{array}$ & $\begin{array}{c}\text { No. }(\%) \text { of } \\
\text { evaluated } \\
\text { outbreaks }\end{array}$ \\
\hline Norovirus & $45(42)$ & $10(45)$ \\
Salmonella & $12(11)$ & $3(14)$ \\
Clostridium perfringens & $9(8)$ & $3(14)$ \\
Scombrotoxin & $4(4)$ & $1(5)$ \\
Campylobacter & $3(3)$ & $1(5)$ \\
Other & $9(9)$ & 0 \\
Unknown & $25(23)$ & $4(18)$ \\
Total & $107(100)$ & $22(100)$ \\
\hline
\end{tabular}

${ }^{a}$ Confirmed foodborne outbreaks in restaurants in EHS-Net catchment area with onset between June 2002 and June 2003, as reported to the Electronic Foodborne Outbreak Reporting System.

next most common agents identified, together accounting for $19 \%$ of all restaurant outbreaks and $28 \%$ of the evaluated outbreaks. Outbreaks ranged in size from 2 to 250 cases, with a median of 15 cases for outbreaks that were evaluated and 8 for outbreaks that were not $(P=0.17)$. Thirteen evaluated outbreaks (59\%) involved 10 or more cases.

Contributing factors identified during outbreak investigations and reported to EFORS reflected the preponderance of outbreaks caused by noroviruses (Fig. 1). For 65\% of outbreaks, handling of food by an infected person or carrier was identified as a contributing factor. For $35 \%$ of outbreaks, bare-hand contact with food was identified as a contributing factor. These two factors were the only contributing factors identified in more than $20 \%$ of the outbreak investigations.

A total of 347 nonoutbreak restaurant evaluations were conducted. Many of the characteristics of the outbreak restaurants were similar to those of the nonoutbreak restaurants. Twelve outbreak restaurants (55\%) and 170 nonoutbreak restaurants (49\%) were operated as parts of a regional or national chain. Outbreak and nonoutbreak establishments were similar with respect to the number of meals served, with a median range for both groups of 200 to 399 meals per week. Training for food workers was offered in $95 \%$ of outbreak and $93 \%$ of nonoutbreak establishments. In both settings, the predominant mode of training offered was on the job, as reported by $81 \%$ of outbreak restaurants and $77 \%$ of nonoutbreak restaurants that offered food worker training. Possible language barriers, which occurred when the primary language of the food workers was not spoken by the manager, were reported in $27 \%$ of outbreak and $35 \%$ of nonoutbreak restaurants. Outbreak and nonoutbreak restaurants also were similar with respect to policies, such as providing sick leave benefits to food workers, requiring food workers to report illnesses to a manager, and restrictions on ill food workers (Table 2).

Outbreak and nonoutbreak establishments differed in several respects. Restaurant characteristics were associated with an apparent increased risk for outbreaks. Nineteen out- 


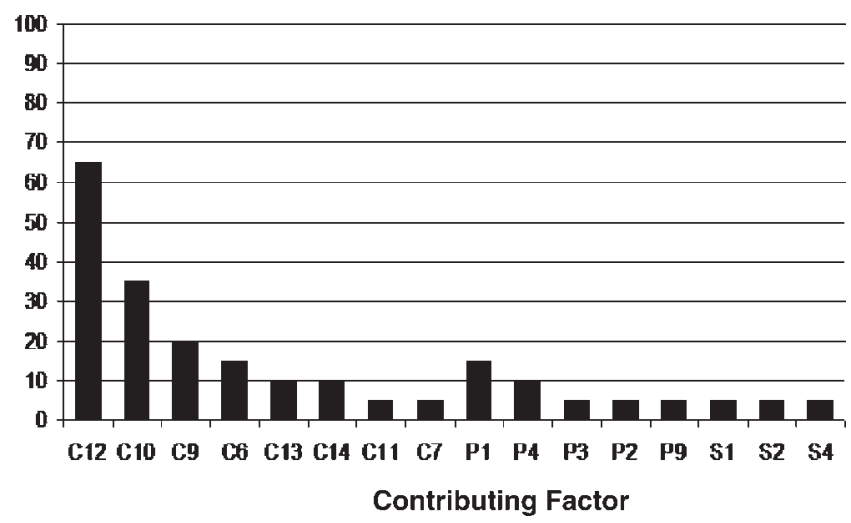

FIGURE 1. Percentage of outbreaks in restaurants with specific contributing factors identified and reported on standardized reporting forms to the CDC Electronic Foodborne Outbreak Reporting System and validated by EHS-Net outbreak evaluation, EHS-Net, 2002 and 2003. Factors C1 through C14 concern contamination of implicated food items, factors P1 through P9 concern conditions that would allow bacterial proliferation on contaminated food items, and factors S1 through S4 concern conditions that would allow survival of microbial agents on contaminated food items. C12, handling by infected person or carrier; C10, bare-hand contact; C9, cross-contamination from raw ingredient; C6, raw product or ingredient of animal origin; C13, inadequate cleaning of equipment; C14, gloved-hand contact; C11, storage in contaminated environment; $C 7$, ingestion of contaminated raw product; $P 1$, foods at room temperature for several hours; P4, preparing foods a half-day or more before serving; P2, slow cooling; P3, inadequate cold-holding temperatures; P9, inadequate thawing; S1, insufficient time or temperature during initial cooking; S2, insufficient time or temperature during reheating; S4, insufficient thawing or insufficient cooking.

break establishments (86\%) and 194 nonoutbreak establishments $(56 \%)$ were regular sit-down restaurants (OR, 5.0; $95 \%$ CI, 1.4 to 21.6$)$. Ten outbreaks $(46 \%)$ were reported in ethnic restaurants; however, only $20 \%$ of the nonoutbreak restaurant evaluations included ethnic restaurants (OR, 3.3; 95\% CI, 1.3 to 8.5).

Certified kitchen managers (CKM) were managers who received a certificate upon completion of a food safety training course. Although details of these courses were not identified, CKMs were associated with a reduced risk for an outbreak. Only $32 \%$ of outbreak restaurants had a CKM,
TABLE 2. Comparison of characteristics for outbreak and nonoutbreak restaurants evaluated by EHS-Net, June 2002 through June 2003

\begin{tabular}{lccc}
\hline & No. (\%) of restaurants & \\
\cline { 2 - 3 } \multicolumn{1}{c}{ Characteristic } & Outbreak & $\begin{array}{c}\text { Nonout- } \\
\text { break }\end{array}$ & $\begin{array}{c}\text { Odds ratio } \\
(95 \% \text { CI })\end{array}$ \\
\hline $\begin{array}{l}\text { Restaurant has certified } \\
\text { kitchen manager }\end{array}$ & $7(32)$ & $243(71)$ & $0.2(0.1-0.5)$ \\
$\begin{array}{l}\text { Manager is aware of haz- } \\
\text { ard analysis critical } \\
\text { control points }\end{array}$ & $10(46)$ & $221(64)$ & $0.5(0.2-1.2)$ \\
$\begin{array}{l}\text { Restaurant offers sick } \\
\text { leave benefits for food } \\
\text { workers }\end{array}$ & $8(35)$ & $62(18)$ & $2.6(0.95-7.0)$ \\
$\begin{array}{l}\text { Food workers are re- } \\
\text { quired to report illness } \\
\text { to a manager }\end{array}$ & $17(77)$ & $289(84)$ & $0.6(0.2-2.1)$ \\
$\begin{array}{l}\text { Ill food workers are re- } \\
\text { stricted from working }\end{array}$ & $16(73)$ & $286(86)$ & $0.5(0.2-1.4)$ \\
\hline
\end{tabular}

compared with $71 \%$ of nonoutbreak restaurants (OR, 0.2; 95\% CI, 0.1 to 0.5 ). The protective effect of a CKM appeared to be due to multiple mechanisms. For example, none of the three outbreaks caused by $C$. perfringens and only 2 of 10 outbreaks caused by a norovirus occurred in restaurants with CKMs. Bare-hand contact with food was not identified in any of the outbreaks where a restaurant had a CKM but was identified as a contributing factor in 7 (47\%) of 15 outbreak restaurants without a CKM. All seven CKMs were familiar with the concept of hazard analysis critical control point (HACCP), a systematic approach in identifying, evaluating, and controlling food safety hazards. Only $3(20 \%)$ of 15 managers who were not certified were familiar with HACCP $(P<0.001)$. However, awareness of HACCP was not independently associated with outbreak status (Table 2).

In contrast to these findings, neither the presence of a CKM nor the presence of policies regarding employee health significantly affected the identification of an infected person or carrier as a contributing factor (Table 3). Most restaurants did not offer sick leave benefits for food workers but did require food workers to report illnesses to a

TABLE 3. Food handling by infected person or carrier (factor C12) identified as a contributing factor in outbreak evaluation by EHS-Net, June 2002 through June 2003

\begin{tabular}{|c|c|c|c|c|c|}
\hline \multirow[b]{2}{*}{ Characteristic } & \multicolumn{2}{|c|}{$\begin{array}{l}\text { No. of evaluated } \\
\text { restaurants }\end{array}$} & \multicolumn{2}{|c|}{$\begin{array}{l}\text { No. }(\%) \text { of restaurants } \\
\text { C12 identified }\end{array}$} & \multirow{2}{*}{$\begin{array}{l}\text { Odds ratio } \\
(95 \% \mathrm{CI})\end{array}$} \\
\hline & Yes & No & Yes & No & \\
\hline \multicolumn{6}{|l|}{ Restaurant has certified kitchen } \\
\hline manager & 5 & 15 & $3(60)$ & $10(67)$ & $0.8(0.1-11.9)$ \\
\hline Manager is aware of HACCP & 8 & 15 & $6(75)$ & $7(47)$ & $3.4(0.4-43.3)$ \\
\hline $\begin{array}{l}\text { Restaurant offers sick leave benefits for food } \\
\text { workers }\end{array}$ & 5 & 14 & $3(60)$ & $10(71)$ & $0.6(0.1-10.1)$ \\
\hline $\begin{array}{l}\text { Food workers are required to report illness to a } \\
\text { manager }\end{array}$ & 15 & 5 & $11(73)$ & $2(40)$ & $4.1(0.3-62.4)$ \\
\hline Ill food workers are restricted from working & 15 & 5 & $11(73)$ & $2(40)$ & $4.1(0.3-62.4)$ \\
\hline
\end{tabular}


TABLE 4. Comparison of kitchen manager training for outbreak and nonoutbreak restaurants evaluated by EHS-Net, June 2002 through June 2003

\begin{tabular}{lccl}
\hline \multirow{2}{*}{$\begin{array}{c}\text { Kitchen manager } \\
\text { certification training }\end{array}$} & No. $(\%)$ of restaurants & \\
\cline { 2 - 3 } & Outbreak & $\begin{array}{c}\text { Nonout- } \\
\text { break }\end{array}$ & $\begin{array}{c}\text { Odds ratio } \\
(95 \% \text { CI })\end{array}$ \\
\hline $\begin{array}{l}\text { State or local agency } \\
\text { Private organization }\end{array}$ & $1(5)$ & $86(25)$ & $0.1(0.0-0.6)$ \\
$\begin{array}{l}\text { Restaurant corporation } \\
\text { or company }\end{array}$ & $3(27)$ & $97(28)$ & $0.4(0.1-1.2)$ \\
Manager not certified & $15(68)$ & $125(36)$ & $0.2(0.03-0.6)$ \\
\hline
\end{tabular}

${ }^{a}$ Categories for certification training are not mutually exclusive.

manager and restricted ill food workers from working. Infected persons or carriers were as likely to be cited as contamination factors in outbreaks in restaurants with these policies as without. In only one outbreak did the manager describe a policy of asking ill food workers whether they had diarrhea; an infected person or carrier was not identified as a contributing factor in that restaurant.

Food safety certification training provided to kitchen managers from a state or local agency or a restaurant corporation was associated with a reduced likelihood of outbreak in the restaurant (Table 4).

\section{DISCUSSION}

The results of these first systematic environmental evaluations conducted by EHS-Net suggest that the presence of a CKM reduces the risk for an outbreak and was the major distinguishing factor between the outbreak and nonoutbreak restaurants. In particular, CKMs were associated with the absence of bare-hand contact with food as a contributing factor and with fewer norovirus- and C. perfringens-associated outbreaks. These findings suggest that CKM awareness of HACCP may have resulted in more effective control of bare-hand contact with ready-to-eat foods and food temperatures during cooling and reheating. Better control of these hazards may have affected the distribution of outbreaks by type of agent. Because most restaurants appeared to rely on on-the-job food safety training for food workers, CKMs likely improved the quality of this training.

During the study period, $60 \%$ of foodborne outbreaks in EHS-Net sites occurred in restaurants. Although there may be a bias toward detecting and investigating outbreaks associated with restaurants, this bias is unlikely to have influenced the results of the outbreak evaluations with respect to food safety practices. The outbreaks evaluated for this study appeared to broadly represent all outbreaks reported during the study period with respect to etiology. In particular, noroviruses were the most common agent identified. Consistent with the epidemiology of noroviruses, the presence of ill food handlers and bare-hand contact with ready-to-eat foods were the most frequently reported contributing factors. However, neither the presence of a CKM nor the presence of policies providing sick leave for food workers, reporting of employee illness, and restriction of ill food workers appeared to reduce the role of ill food handlers as a major contamination source leading to outbreaks. These findings suggest that (i) food workers do not understand the importance of remaining away from work while ill with vomiting or diarrhea, (ii) illnesses among food workers are not being effectively monitored, or (iii) commitment is lacking to enforce policies regarding ill food workers. Results of this study suggest that the importance of managing food worker illnesses must be emphasized during food safety training programs.

The importance of managing food worker illnesses can be emphasized further by estimating the number of food workers who are infected by noroviruses but who work while they are ill. Such a projection can be made using the National Restaurant Association's estimate of 12.2 million persons employed in restaurants in the United States (13). Using estimates from Mead and colleagues (11) of the frequency of acute diarrheal illness in the general population ( 0.79 episodes per person per year) and the proportion attributable to norovirus (11\%), as many as 1 million restaurant employees per year may be infected with noroviruses. In an EHS-Net study of food workers interviewed during FoodNet population surveys, $5 \%$ of food workers admitted to working while ill with vomiting or diarrhea (4). Thus, 50,000 norovirus-infected U.S. food workers are likely to work while ill.

Certain characteristics of restaurants, including those with regular sit-down table service and ethnic menus, appeared to be associated with outbreaks. However, each also represents a readily identifiable feature of a restaurant that could bias the likelihood that an outbreak would be detected, reported, or evaluated. The sit-down table service format was highly correlated with complex meal service, which was used as one of the selection criteria for nonoutbreak restaurant evaluations. Thus, the actual significance of these findings is uncertain. In contrast, the presence of a CKM in the restaurant should not have been subject to detection, reporting, or evaluation biases.

In previous studies, researchers have attempted to associate routine restaurant inspection scores with subsequent foodborne illnesses $(1,2,6,12)$. In Seattle-King County during 1986 and 1987, restaurants where an outbreak occurred had significantly lower mean inspection scores than did matched control restaurants; violations of temperature control of potentially hazardous foods were most strongly associated with outbreaks (6). During the mid-1990s, studies conducted in Miami-Dade County and Los Angeles County involved similar methods $(1,2)$. In Los Angeles, investigated foodborne incidents were associated with incorrect storage of food, reuse of food, and any food protection violation (1). In Miami-Dade County, the presence of vermin was associated with outbreaks (2). Restaurant size was the only factor consistently associated with case status in all three studies; larger restaurants had more foodborne disease incidents. However, the size of reported outbreaks in these retrospective records-based studies was small (most involved $<10$ cases), and the etiologic agent was not determined for most of the outbreaks. Thus, uncertainty about the relation between the inspection results 
and the corresponding illness events (8) may explain in part the lack of consistent results, except for restaurant size, across the studies. More recently, Jones et al. (9) found no difference in mean inspection scores for restaurants preceding an outbreak compared with all restaurant inspections in Tennessee from 1999 through 2002.

In Scotland during nationwide outbreaks of infection caused by Salmonella serotype Enteritidis associated with eating in restaurants, no differences in inspection scores were observed between restaurants where affected individuals reported eating compared with restaurants where no affected individuals reported eating (12). This finding suggests that routine food handling practices may have allowed foodborne disease transmission. For example, an EHS-Net study of egg-preparation practices in the United States revealed that $54 \%$ of restaurants that prepared breakfast egg entrees pooled raw shell eggs not intended for immediate service (10). The risk for foodborne illness depends both on the presence of a specific source of contamination and food handling practices that allow its transmission.

The failure in previous studies to link inspection results with outbreaks also reflects the limitations of routine inspections for identifying and changing the underlying factors that lead to outbreaks. Traditionally, restaurant inspections have provided point-in-time compilations of readily observable food handling and sanitation deficiencies. These "snapshots" lead restaurant managers to focus on fixing specific violations rather than on evaluating the overall performance of their food safety systems. In contrast, the EHSNet environmental evaluations were designed to assess food preparation as a system and to identify both the system failures that led to the outbreak and the underlying reason for their occurrence. For example, the relationship between ill food handlers and bare-hand contact with ready-to-eat foods as a contributing factor and the presence of a CKM as a mitigating factor has important implications for the role of management in maintaining food safety.

Unfortunately, the small number of outbreak evaluations conducted during this study period made it impossible to fully develop multivariate models for comparing environmental evaluations between outbreak and nonoutbreak settings. Thus, our outbreak and nonoutbreak analyses were primarily limited to establishment characteristics. However, on the basis of the contamination factors identified in these outbreaks, we separately examined factors associated with good food handling practices in restaurants that have not experienced a foodborne outbreak. Nonoutbreak restaurant evaluations such as these can provide critical insights concerning the role of the CKM in promoting good food handling practices and ways to improve the relevance of routine restaurant inspections.

Although the results of our evaluations suggest that the presence of a CKM and control of bare-hand contact with foods may help prevent noroviral disease outbreaks, the key determinant appears to be the presence of an infected food worker. This conclusion must be confirmed by further studies involving a larger series of outbreaks. Clarification of the important role of infected restaurant workers in foodborne disease outbreaks, particularly those associated with noroviruses, will have important implications for food safety training and disease prevention.

\section{ACKNOWLEDGMENTS}

The authors thank the following: Fred Angulo (National Center for Infectious Disease, CDC) and Shirley Bohm, Faye Feldstein, John Guzewich, Thomas Hill, Glenda Lewis, Patrick McCarthy, and Eileen Parish (Center for Food Safety and Applied Nutrition, U.S. Food and Drug Administration) for their assistance with the development of evaluation questions; Curtis Blanton (National Center for Environmental Health, CDC) for his assistance with statistical analysis; Scott Brown (National Center for Environmental Health, CDC), for developing the EHS-Net information system; and the following EHS-Net specialists for data collection Peter Esko (California Department of Health), Michelle Motsinger (Colorado Department of Public Health), Tracy Weeks (Connecticut Department of Public Health), Tammi Stigger (Georgia Department of Human Resources), Dave Riemann, April Bogard, and Pete Giesen (Minnesota Department of Health), James Mack (Oregon Health Services), Ray Woron (New York Department of Health), and Danny Ripley (Metro Public Health Department, Nashville, Tenn.). The members of the EHS-Net Working Group are Peter Esko, Michelle Motsinger, Tracey Weeks, Lisa Bushnell (Connecticut Department of Public Health), Tammi Stigger, Melissa Tobin D'Angelo (Georgia Department of Human Resources), April Bogard, Steven Diaz (Minnesota Department of Health), Pete Giesen, David Riemann, David Nicholas (New York Department of Health), Ray Woron, James Mack, Lore E. Lee (Oregon Health Services), Ryan Mason (Metro Public Health Department, Nashville), Danny Ripley, and Curtis Blanton, Scott Brown, Laura Green, Craig Hedberg, Rhonda Jaster, Jennifer Keagy, Elizabeth Kirkland, John Li, Latoria Whitehead, Vince Radke, and Carol Selman (all from the National Center for Environmental Health, CDC).

\section{REFERENCES}

1. Buchholz, U., G. Run, J. L. Kool, J. Fielding, and L. Mascola. 2002. A risk-based restaurant inspection system in Los Angeles County. J. Food Prot. 65:367-372.

2. Cruz, M. A., D. J. Katz, and J. A. Suarez. 2001. An assessment of the ability of routine restaurant inspections to predict foodborne outbreaks in Miami-Dade County, Florida. Am. J. Public Health 91: 821-823.

3. Gelting, R., J. Sarisky, C. Selman, C. Otto, C. Higgins, P. Bohan, S. Buchanan, and P. Meehan. 2005. Use of a systems-based approach to an environmental health assessment for a waterborne disease investigation at a snowmobile lodge in Wyoming. Int. J. Hyg. Environ. Health 208(1-2):67-73.

4. Green, L., C. Selman, A. Banerjee, R. Marcus, C. Medus, F. J. Angulo, V. Radke, S. Buchanan, and the EHS-Net Working Group. 2005. Food service workers' self-reported food preparation practices: an EHS-Net study. Int. J. Hyg. Environ. Health 208(1-2):27-35.

5. Higgins, C., and B. Hartfield. 2004. A systems-based food safety evaluation: an experimental approach. J. Environ. Health 67(4):914.

6. Irwin, K., J. Ballard, J. Grendon, and J. Kobayashi. 1989. Results of routine restaurant inspections can predict outbreaks of foodborne illness: the Seattle-King County experience. Am. J. Public Health 79:586-590.

7. Jones, T., D. Vugia, C. Selman, F. Angulo, and the EIP FoodNet Working Group. 2002. Eating in restaurants: a risk factor for foodborne illness? Findings from FoodNet to be explored by EHS-Net. Presented at the International Conference on Emerging Infectious Diseases, Atlanta, March 2002.

8. Jones, T. F., B. Imhoff, M. Samuel, P. Mshar, P. G. McCombs, M. Hawkins, V. Deneen, M. Cambridge, and S. J. Olsen. 2004. Limitations to successful investigation and reporting of foodborne outbreaks: an analysis of foodborne disease outbreaks in FoodNet catchment areas, 1998-1999. Clin. Infect. Dis. 38(Suppl. 3):S297-S302.

9. Jones, T. F., B. I. Pavlin, B. J. LaFleur, L. A. Ingram, and W. Schaffner. 2004. Restaurant inspection scores and foodborne disease. Emerg. Infect. Dis. 10:688-692. 
10. Lee, R., M. E. Beatty, A. K. Bogard, M. P. Esko, F. J. Angulo, C. Selman, and the EHS-Net Working Group. 2004. Prevalence of highrisk egg-preparation practices in restaurants that prepare breakfast egg entrees: an EHS-Net study. J. Food Prot. 67:1444-1450.

11. Mead, P. M., L. Slutsker, V. Dietz, L. F. McCaig, J. S. Bresee, C. Shapiro, P. M. Griffin, and R. V. Tauxe. 1999. Food-related illness and death in the United States. Emerg. Infect. Dis. 5:607-625.

12. Mullen, L. A., J. M. Cowden, D. Cowden, and R. Wong. 2002. An evaluation of the risk assessment method used by environmental health officers when inspecting food businesses. Int. J. Environ. Health Res. 12:255-260.

13. National Restaurant Association. 2005. Restaurant industry 2005 fact sheet. National Restaurant Association, Washington, D.C. Available at: http://www.restaurant.org/pdfs/research/2005factsheet.pdf. Accessed 19 July 2005.

14. Olsen, S. J., L. C. MacKinnon, J. S. Goulding, N. H. Bean, and L. Slutsker. 2000. Surveillance for foodborne-disease outbreaks, United States, 1993-1997. Morb. Mortal. Wkly. Rep. 49(SS-1):1-51. 